\title{
Efecto Antiangiogénico de la Triamcinolona Asociada a Lidocaína con Epinefrina de Uso Odontológico en la Membrana Alantocoriónica de Pollo
}

\author{
Antiangiogenic Effect of Triamcinolone Associated with Lidocaine with \\ Epinephrine for Dental use in Chicken Chorioallantoic Membrane
}

\author{
Marcelo Mardones M. ${ }^{1}$; Rodrigo Bravo A. ${ }^{1}$; Luis A. Córdova ${ }^{\mathbf{1}}$; \\ Josefina Santana L. ${ }^{2}$; Claudio Zamorano C. ${ }^{3}$ \& David Lemus A. ${ }^{4}$
}

\begin{abstract}
MARDONES, M. M.; BRAVO, A. R.; CÓRDOVA, L. A.; SANTANA, L. J.; ZAMORANO, C. C. \& LEMUS, A. D. Efecto antiangiogénico de la triamcinolona asociada a lidocaína con epinefrina de uso odontológico en la membrana alantocoriónica de pollo. Int. J. Morphol., 37(1):344-348, 2019.
\end{abstract}

RESUMEN: El proceso angiogénico se define como el proceso en el que los vasos sanguíneos generan brotes dando como resultado neovascularidad. Un desbalance en el proceso angiogénico contribuye a numerosos desórdenes inflamatorios, infecciosos, isquémicos, inmunológicos y malignos. En el territorio maxilofacial se pueden encontrar patologías neoplásicas benignas de desarrollo local con un marcado componente angiogénico que determinan su crecimiento y agresividad. Sin embargo, existe escasa evidencia de cómo tratarlas en base al control de la angiogénesis. Terry \& Jacoway (1994) desarrollaron un protocolo de tratamiento para lesiones neoplásicas benignas con un importante componente vascular que se utiliza actualmente. Este protocolo consiste en la infiltración intralesional de una suspensión de triamcinolona $10 \mathrm{mg} / \mathrm{ml}$ más una solución de anestésico local de uso odontológico como la lidocaína al $2 \%$ asociada a epinefrina en una concentración de 1:200.000. Sin embargo, el uso de epinefrina podría disminuir la acción antiangiogénica de la triamcinolona al ser un vasoconstrictor. El objetivo de este estudio es comparar el efecto antiangiogénico, en la membrana alantocoriónica de pollo (MAC), de esta suspensión versus el efecto de la triamcinolona sin asociar a anestésicos locales. Los resultados del efecto antiangiogénico en la MAC de pollo, obtenidos en la investigación concluyeron que la suspensión de triamcinolona asociada a lidocaína con epinefrina es similar al de la suspensión de triamcinolona sin asociar a anestésicos locales. Además, se logró determinar que las suspensiones de triamcinolona sin asociar a anestésicos locales y las asociadas a anestésicos locales con o sin vasoconstrictor poseen un marcado efecto antiangiogénico, en la MAC de pollo, en comparación al grupo control.

PALABRAS CLAVE: Angiogénesis; Membrana alantocoriónica; Triamcinolona; Epinefrina.

\section{INTRODUCCIÓN}

La Angiogénesis o Neovascularización, es un proceso en el que los vasos sanguíneos formados generan brotes capaces de formar nuevos vasos (Hillen\& Griffioen, 2007); siendo un evento fisiológico importante, involucrado en diferentes procesos como la inflamación crónica, cicatrización, ovulación y la formación placentaria. Cuando se produce un aumento de la angiogénesis en tejidos alterados, estos producen cantidades anormales de factores de crecimiento angiogénico (Motamed \& Sage, 1997), sobrepasando la capacidad de regulación de inhibidores naturales de la angiogénesis e implica la destrucción de los tejidos normales como es el caso de las neoplasias. Los tumores primarios necesitan de los capilares sanguíneos para poder crecer y diseminarse en forma metastásica; así, la angiogénesis es una etapa imprescindible para la progresión de la neoplasia.

Independientemente del mecanismo de acción de una droga antiangiogénica, todas ellas incrementan los porcentajes de apoptosis de las células tumorales. Probablemente entonces, la apoptosis es inducida por la hipoxia tumoral creada al inhibirse el proceso de angiogénesis tumoral. Esto significa que el número de vasos tumorales que pueden mantenerse o crecer, a pesar del tratamiento angiostático, determinará al número de células tumorales que sobrevivirán al

\footnotetext{
${ }^{1}$ Cirujano Máxilo Facial Hospital San José, Santiago, Chile. Profesor Asistente, Facultad Odontología, Universidad de Chile, Santiago, Chile.

${ }^{2}$ Residente de Cirugía y Traumatología Buco Máxilo Facial de la Universidad de Chile, Santiago, Chile.

${ }^{3}$ Residente Estadía Perfeccionamiento Equipo Cirugía Máxilo Facial, Hospital San José, Santiago, Chile.

${ }^{3}$ Programa de Anatomía y Biología del Desarrollo. Facultad de Medicina, Universidad de Chile, Santiago, Chile.
} 
MARDONES, M. M.; BRAVO, A. R.; CÓRDOVA, L. A.; SANTANA, L. J.; ZAMORANO, C. C. \& LEMUS, A. D. Efecto antiangiogénico de la triamcinolona asociada a lidocaína con epinefrina de uso odontológico en la membrana alantocoriónica de pollo. Int. J. Morphol., 37(1):344-348, 2019.

tratamiento y estas determinarán el tamaño tumoral (Aravind et al., 1999). Se han identificado diferentes factores angiostáticos o antiangiogénicos, de los cuales, los corticoides han sido ampliamente estudiados, entre ellos la acción de la betametasona, dexametasona (Folkman \& Ingber, 1987) y triamcinolona. El acetato de triamcinolona es un corticoide de gran potencia antinflamatoria que disminuye la secreción de IL-6 y del VEGF teniendo un efecto antiangiogénico marcado en la neovascularización (Ebrahem et al., 2006; Geltzer et al., 2013).

En el territorio maxilofacial se pueden presentar neoplasias benignas que generalmente no provocan metástasis a distancia, pero que son agresivas localmente, cuyo crecimiento es muy marcado, destruyendo estructuras anatómicas adyacentes provocando alteraciones funcionales y morfológicas en el paciente. Algunas de estas lesiones benignas con un marcado componente vascular son: lesiones de células gigantes, lesiones quísticas aneurismáticas, hemangiomas, angioblastomas de células gigantes, entre otras. El tratamiento de este tipo de lesiones, generalmente corresponde a la exéresis quirúrgica de la lesión, que normalmente por el tamaño y compromiso de estructuras adyacentes, se transforma en un tratamiento con una alta morbilidad local, afectando directamente la funcionalidad y morfología facial. Actualmente, existen reportes en la literatura del uso de fármacos locales, como alternativa o complemento al tratamiento quirúrgico, con el objeto de disminuir el tamaño o incluso remitir por completo este tipo de lesiones (Carlos \& Sedano, 2002; de Lange et al., 2007).

Para lesiones con un gran componente vascular que requieran terapia con corticoides, existe un protocolo utilizado en la actualidad (Adornato \& Paticoff, 2001; Graham et al., 2008; Mohanty \& Jhamb, 2009; Nogueira et al, 2010; Ferretti \& Muthray, 2011), descrito por Terry \& Jacoway (1994), que consiste en infiltraciones intralesionales de una suspensión de triamcinolona $10 \mathrm{mg} / \mathrm{ml}$ asociada a lidocaína al $2 \%$ (anestésico local) con un vasoconstrictor como la epinefrina en una concentración de 1:200.000. En una proporción de 1:1, infiltrando 1 centímetro cúbico (cc) de suspensión por cada cc de lesión, sugiriendo una infiltración semanal por un período de 6 semanas.

Sin embargo, el uso de epinefrina como vasoconstrictor tiene ciertas restricciones que deben considerarse al momento de indicar una terapia infiltrativa intralesional. Las interacciones con otras drogas como beta bloqueadores no selectivos y antidepresivos tricíclicos; enfermedades cardiovasculares y antecedentes recientes de infarto al miocardio, son situaciones que deben restringir el uso de un anestésico local asociado a un vasoconstrictor por las posibles complicaciones sistémicas que se puedan oca- sionar. También se ha reportado que el aumento de niveles de epinefrina y norepinefrina tendrían un efecto proangiogénico tumoral, debido a la activación de receptores $b$ adrenérgicos y a la síntesis de factores que favorecen la angiogénesis, aunque estos hallazgos se han encontrado en individuos bajo estrés crónico.

El objetivo de este estudio es comparar el efecto antiangiogénico de una suspensión de triamcinolona asociada a lidocaína al $2 \%$ con epinefrina 1:200.000 con una suspensión de triamcinolona sin asociar a anestésicos locales en la membrana alantocoriónica (MAC) de pollo.

\section{MATERIAL Y MÉTODO}

El estudio experimental se realizó en el Laboratorio de Embriología Experimental del Programa de Morfología (ICBM) de la Facultad de Medicina de la Universidad de Chile. Se ocuparon MAC de pollo, en huevos fecundados de Gallina White Leghorn, adquiridos en el Instituto de Salud Pública (ISP). Estos se incubaron a $38,2^{\circ} \mathrm{C}$, en atmósfera húmeda.

A las 48 horas de incubación, previa desinfección con alcohol yodado al $1 \%$, se realizaron ventanas en las cáscaras y la extracción de $2 \mathrm{ml}$ de albúmina, de modo que la MAC en desarrollo se separara de la cáscara. Luego se sellaron con cinta adhesiva transparente.

A los 8 días se implantó un filtro de metilcelulosa, de $5 \mathrm{~mm}$ de diámetro con poros de $0,25 \mathrm{~mm}$, esterilizado, sobre la superficie de la MAC, con el fin de inducir angiogénesis inflamatoria (Fig. 1A).

A los 10-11 días, sobre los filtros de metilcelulosa, se instilaron las siguientes soluciones: Grupo 1 (Control) $(\mathrm{n}=4)$ : $10 \mathrm{~mL}$ de suero fisiológico; Grupo $2(\mathrm{n}=4): 10 \mathrm{~mL}$ de suspensión de triamcinolona $10 \mathrm{mg}$ más lidocaína al $2 \%$ con epinefrina 1:200.000, diluida en suero fisiológico 1:50; Grupo $3(\mathrm{n}=4): 10 \mathrm{~mL}$ de triamcinolona $10 \mathrm{mg}$ sin asociar a anestésicos locales, diluida en suero fisiológico 1:50; Grupo $4(\mathrm{n}=4)$ : $10 \mathrm{~mL}$ de suspensión triamcinolona $10 \mathrm{mg}$ más lidocaína al $2 \%$ sin vasoconstrictor, diluida en suero fisiológico 1:50 (Fig. 1B).

A los 12-13 días, se realizó la toma y exéresis de las MAC, y fueron procesadas para luego someterlas a tinción de Hematoxilina-Eosina (Fig. 1C).

El recuento de vasos sanguíneos (vs) se realizó mediante observación microscópica óptica a un aumento de 

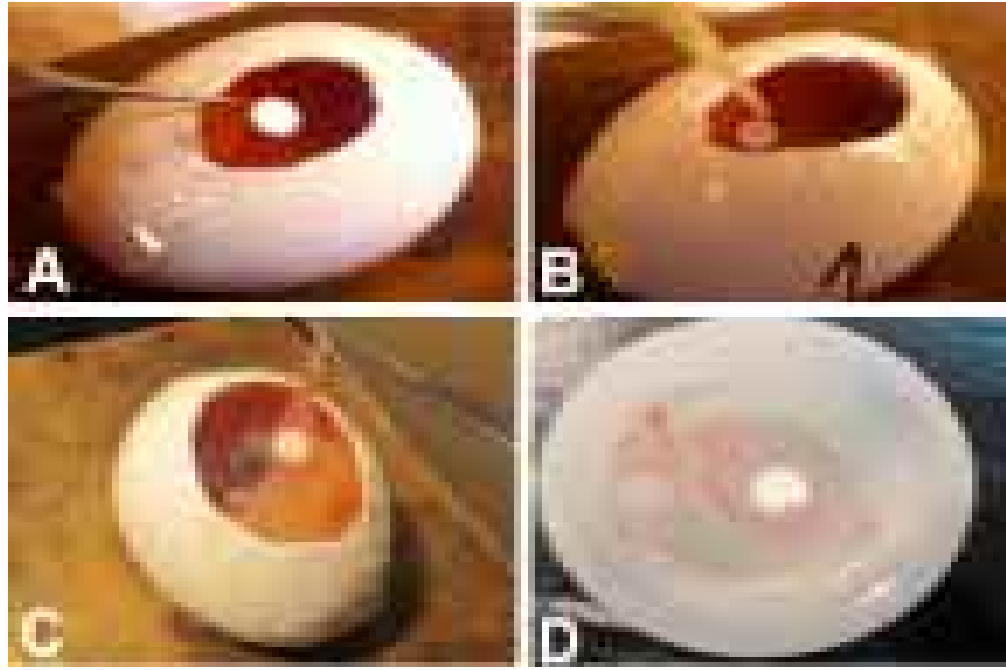

Fig. 1. A. Implantación del filtro de metilcelulosa. B. Instilación de $10 \mathrm{~mL}$ de suspensión en el filtro de metilcelulosa. C. Toma y exéresis de la MAC. D. Fijación de las membranas en alcohol al $100 \%$.

400X, por un operador único debidamente entrenado. Se efectuaron conteos de vasos sanguíneos por campo estereográfico, con un total de 512 lecturas de campo para cada grupo de estudio, obteniéndose valores muestrales del número de vasos sanguíneos.

Se utilizó el T TEST para comparar los promedios de cada grupo experimental entre sí. Se consideró un valor de $\mathrm{P}<0,05$ estadísticamente significativo. El test mencionado fue realizado mediante el software GraphPad InStat @ por un profesional capacitado en bioestadística.

\section{RESULTADOS}

El recuento de vasos sanguíneos obtenidos en los grupos experimentales con las suspensiones estudiadas, fue significativamente menor en comparación a los grupos controles (Fig. 2).

El recuento total de vasos sanguíneos en los 512 campos de lectura por cada grupo de estudio en las MAC de pollo, dieron los siguientes resultados: Grupo 1= 949 vs; Grupo $2=325$ vs; Grupo 3= 314 vs; Grupo 4=412 vs. Con un promedio por placa de cada grupo de estudio (Tabla I; Fig. 3A): Grupo 1= 14,83 vs; Grupo $2=5,16$ vs; Grupo 3= 4,91 vs; Grupo 4= 6,44 vs.

Realizado el $\mathrm{T}$ test entre promedios para los grupos de estudio 1 y 2, dio como resultado un valor de $\mathrm{p}<0,0001$, diferencia estadística altamente significativa (Fig. 3B). Para los grupos de estudio 1 y 3 , dio como resultado un valor de p: 0,0003 ( $p<0,0001)$, diferencia estadística altamente significativa (Fig. 4A); para los grupos de estudio 1 y 4, dio como resultado un valor de $\mathrm{p}: 0,0113$ ( $\mathrm{p}<0,0001)$, diferencia estadística significativa (Fig. 4B). En los grupos de estudio 2 y 3, dio como resultado un valor de p:0,1450 ( $<$ < 0,0001 ) diferencia estadística no significativa (Fig. 5A); y para los grupos de estudio 3 y 4 , dio como resultado un valor de p: 0,1193 ( $<$ 0,0001), diferencia estadística no significativa (Fig. 5B).
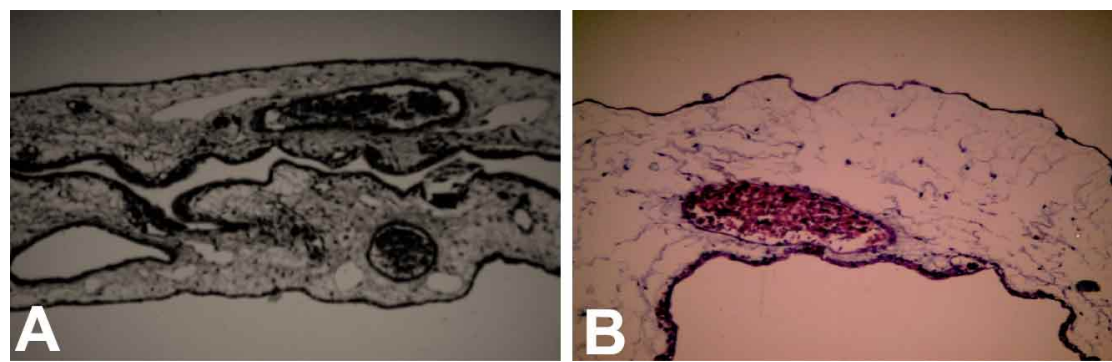

Fig. 2. Visión microscópica óptica 10X de MAC procesadas con tinción de hematoxilinaeosina. A. Grupo 1 (grupo control). B. Grupo 2 (triamcinolona asociada a lidocaína con epinefrina).
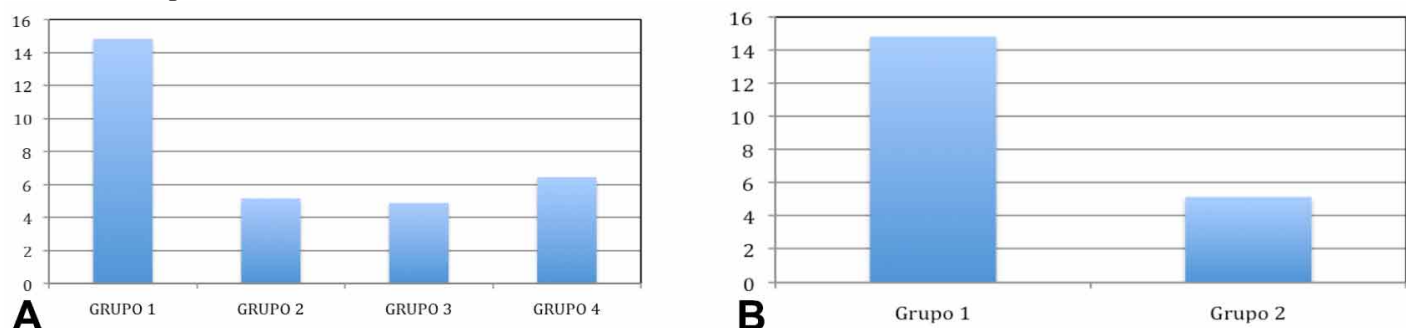

Fig. 3. A. Promedios de vasos sanguíneos por grupo de estudio. B. Promedio vasos sanguíneos grupos de estudio 1 y 2. 

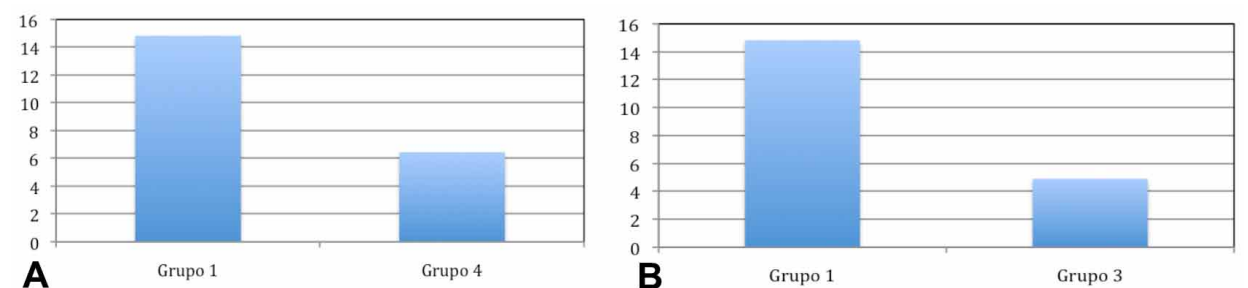

Fig. 4. A. Promedio de vasos sanguíneos grupos de estudio 1 y 3. B. Promedio de vasos sanguíneos grupos de estudio 1 y 4.
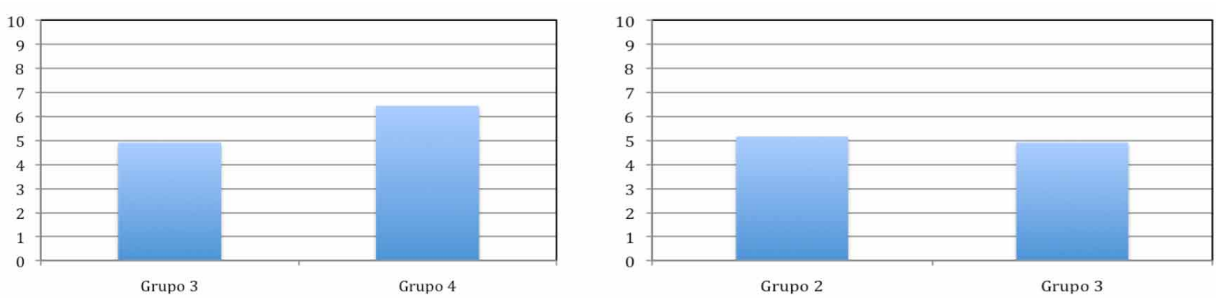

Fig. 5. A. Promedio de vasos sanguíneos grupos de estudio 2 y 3. B. Promedio de vasos sanguíneos grupos de estudio 3 y 4 .

Tabla I. Grupos de estudio y promedio de recuento de vasos sanguíneos.

\begin{tabular}{llccc}
\hline $\begin{array}{l}\text { Grupo de } \\
\text { estudio }\end{array}$ & \multicolumn{1}{c}{ Tratamiento } & $\begin{array}{c}\text { Cantidad } \\
\text { instilada }\end{array}$ & $\begin{array}{c}\text { Número total } \\
\text { de vasos }\end{array}$ & $\begin{array}{c}\text { Promedio de } \\
\text { vasos }\end{array}$ \\
\hline 1 & Suero fisiológico & $10 \mu \mathrm{L}$ & 949 & 14,83 \\
2 & $\begin{array}{l}\text { Triamcinolona }+1 \text { idocaína } \\
\text { con epinefrina }\end{array}$ & $10 \mu \mathrm{L}$ & 325 & 5,16 \\
3 & $\begin{array}{l}\text { Triamcinolona sin asociar a } \\
\text { anestésico local }\end{array}$ & $10 \mu \mathrm{L}$ & 314 & 4,91 \\
4 & $\begin{array}{l}\text { Triamcinolona + lidocaína sin } \\
\text { vasoconstrictor }\end{array}$ & $10 \mu \mathrm{L}$ & 412 & 6,44 \\
\hline
\end{tabular}

\section{DISCUSIÓN}

El estudio del efecto de las suspensiones de triamcinolona en la angiogénesis de la MAC de pollo, tuvo un resultado positivo para evaluar las propiedades antiangiogénicas de estas drogas. La MAC del pollo es un buen modelo de estudio in vivo para estos fines, ya que es una estructura anatómica sencilla de acceder y experimentar, posee un costo moderado y es de fácil replicación (Vargas et al., 2007; Balke et al., 2010).

El efecto antiangiogénico de las suspensiones de triamcinolona con o sin asociación a anestésicos locales tuvo un efecto significativo en el recuento total de vasos sanguíneos en la MAC de pollo comparadas con el grupo control infiltrado con suero fisiológico, confirmando el efecto antiangiogénico que poseen los corticoides en general (Mc Kay, 2008; Banciu et al., 2008).

Sin embargo, la diferencia del efecto antiangiogénico entre la suspensión de triamcinolona sin asociar a anestésicos locales comparada con las que estaban asociadas a anestésicos locales con y sin vasoconstrictor, no produjeron diferencias estadísticas significativas en el recuento total de vasos sanguíneos. Por lo tanto, estos hallazgos nos podrían orientar clínicamente a que como parte del tratamiento antiangiogénico en las patologías neoplásicas benignas con un importante componente vascular en el territorio maxilofacial, bastaría sólo con infiltrar una suspensión de triamcinolona sin asociación a otros medicamentos como los anestésicos locales, o realizar una asociación a éstos fármacos en una proporción menor a la descrita en el protocolo de Terry \& Jacoway, con el fin de mantener los efectos locales deseables del anestésico, como la disminución del dolor post infiltración.

Según el protocolo de Terry \& Jacoway, la suspensión a infiltrar en una lesión, se compone de una asociación de triamcinolona y anestésico local con vasoconstrictor en una proporción de 1:1. La cantidad de infiltración de esta suspensión debe ser $1 \mathrm{cc}$ por cada cc de lesión o por cada cc de 
radiolucidez de la lesión, por lo que la cantidad de vasoconstrictor presente en la suspensión que podría inyectarse en una lesión de mayor tamaño $(>7 \mathrm{~cm})$ podría causar alteraciones cardiovasculares importantes en el paciente, e incluso la epinefrina presente en la solución podría tener un efecto proangiogénico no deseable para el tratamiento de este tipo de lesiones (Chakroborty et al., 2009). También es necesario considerar la propiedad farmacológica vasodilatadora de los anestésicos locales, especialmente la lidocaína, que podría determinar un aumento en el flujo sanguíneo y en la angiogénesis tumoral local.

Con los datos observados en el estudio, la proporción de 1:1, entre la triamcinolona y el anestésico local, podría modificarse disminuyendo la cantidad de anestésico local o incluso suspender este fármaco de la composición de esta suspensión. No obstante, se requieren mayores estudios para determinar el tratamiento ideal con menores efectos secundarios para el paciente que presente este tipo de lesiones y eventualmente generar una modificación al protocolo actual vigente.

MARDONES, M. M.; BRAVO, A. R.; CÓRDOVA, L. A.; SANTANA, L. J.; ZAMORANO, C. C. \& LEMUS, A. D. Antiangiogenic effect of triamcinolone associated with lidocaine with epinephrine for dental use in chicken chorioallantoic membrane. Int. J. Morphol., 37(1):344-348, 2019.

SUMMARY: Angiogenesis is defined as the process through which new blood vessels form from previously existing vessels. Several inflammatory, infectious, ischemic, immunological and malignant disorders are caused by the lack of adequate angiogenesis balance. In the maxillofacial area, there are invasive benign neoplastic pathologies with a strong angiogenic component, which determines aggressive behavior and growth. Studies in the literature are scarce regarding treatment of these conditions based on angiogenesis control. Currently, the protocol used to treat these maxillofacial benign neoplastic lesions, was developed in 1994 by Terry \& Jacoway and has a strong angiogenic component. Consequently lesions are treated via intra-lesion administration of triamcinolone $10 \mathrm{mg} / \mathrm{mL}$, a solution used in dental local anesthetic, such as lidocaine $2 \%$, in conjunction with epinephrine at a concentration of $1: 200,000$. The objective of this study was to compare the antiangiogenic effect of this protocol in chicken chorioallantoic membrane (CAM) without the use of local anesthetic. The results of the antiangiogenic effect in the CAM obtained in this study concluded that the effect of the suspension of triamcinolone associated to lidocaine with epinephrine, is similar to the suspension of triamcinolone without associating local anesthetics. Furthermore, it was determined that suspensions of triamcinolone without local anesthetic, and those associated to local anesthetic with, and without vasoconstrictor have a strong antiangiogenic effect in CAM compared to the control group.

KEY WORDS: Angiogenesis; Chorioallantoic membrane; Triamcinolone; Epinephrine.

\section{REFERENCIAS BIBLIOGRÁFICAS}

Adornato, M. C. \& Paticoff, K. A. Intralesional corticosteroid injection for treatment of central giant-cell granuloma. J. Am. Dent. Assoc., 132(2):18690, 2001.

Aravind, L.; Dixit, V. M. \& Koonin, E. V. The domains of death: evolution of the apoptosis machinery. Trends Biochem. Sci., 24(2):47-53, 1999.

Balke, M.; Neumann, A.; Kersting, C.; Agelopoulus, K.; Gebert, C.; Gosheger, G.; Buerger, H. \& Hagedorn, M. Morphologic characterization of osteosarcoma growth on the chick chorioallantoic membrane. B. M. C. Res. Notes, 3:58, 2010.

Banciu, M.; Schiffelers, R. M.; Metselaar, J. M. \& Storm, G. Utility of targeted glucocorticoids in cancer therapy. J. Liposome Res., 18(1):47-57, 2008.

Carlos, R. \& Sedano, H. O. Intralesional corticosteroids as an alternative treatment for central giant cell granuloma. Oral Surg. Oral Med. Oral Pathol. Oral Radiol. Endod., 93(2):161-6, 2002.

Chakroborty, D.; Sarkar, C.; Basu, B.; Dasgupta, P. S. \& Basu, S. Catecholamines regulate tumor angiogenesis. Cancer Res., 69(9):3727-30, 2009.

de Lange, J.; van den Akker, H. P. \& van den Berg, H. Central giant cell granuloma of the jaw: a review of the literature with emphasis on therapy options. Oral Surg. Oral Med. Oral Pathol. Oral Radiol. Endod., 104(5):603-15, 2007.

Ebrahem, Q.; Minamoto, A.; Hoppe, G.; Anand-Apte, B. \& Sears, J. E. Triamcinolone acetonide inhibits IL-6- and VEGF-induced angiogenesis downstream of the IL-6 and VEGF receptors. Invest. Ophthalmol. Vis. Sci., 47(11):4935-41, 2006.

Ferretti, C. \& Muthray, E. Management of central giant cell granuloma of mandible using intralesional corticosteroids: case report and review of literature. $J$. Oral Maxillofac. Surg., 69(11):2824-9, 2011.

Folkman, J. \& Ingber, D. E. Angiostatic steroids. Method of discovery and mechanism of action. Ann. Surg., 206(3):374-83, 1987.

Geltzer, A.; Turalba, A. \& Vedula, S. S. Surgical implantation of steroids with antiangiogenic characteristics for treating neovascular age-related macular degeneration. Cochrane Database Syst. Rev., (4):CD005022, 2013.

Graham, R. M.; Foster, M. E. \& Richardson, D. An unusual presentation of a central giant cell granuloma and initial treatment with intralesional steroids - A case report and review of the literature. J. Oral Health Community Dent., 2(3):65-9, 2008.

Hillen, F. \& Griffioen, A. W. Tumour vascularization: sprouting angiogenesis and beyond. Cancer Metastasis Rev., 26(3-4):489-502, 2007.

Mohanty, S. \& Jhamb, A. Central giant cell lesion of mandible managed by intralesional triamcinolone injections. A report of two cases and literature review. Med. Oral Patol. Oral Cir. Bucal, 14(2):E98-102, 2009.

Motamed, K. \& Sage, E. H. Regulation of vascular morphogenesis by the matricellular protein SPARC. Kidney Int., 51(5):1383-7, 1997.

Nogueira, R. L.; Teixeira, R. C.; Cavalcante, R. B.; Ribeiro, R. A. \& Rabenhosrt, S. H. Intralesional injection of triamcinolone hexacetonide as an alternative treatment for central giant-cell granuloma in 21 cases. Int. J. Oral Maxillofac. Surg., 39(12):1204-10, 2010.

Terry, B. C. \& Jacoway, J. R. Management of central giant cell lesions. Oral Maxillofac. Surg. Clin. North Am., 6:579-600, 1994.

Vargas, A.; Zeisser-Labouèbe, M.; Lange, N.; Gurny, R. \& Delie, F. The chick embryo and its chorioallantoic membrane (CAM) for the in vivo evaluation of drug delivery systems. Adv. Drug Deliv. Rev., 59(11):1162-76, 2007.

Corresponding author:

Marcelo Mardones M.

Cirujano Máxilo Facial

Profesor Asistente

Fac. de OdontologíaUniversidad de Chile

Hospital San José

Santiago - CHILE

Received: 11-07-2018

Accepted: 16-10-2018

Email: drmardones@gmail.com 